Correction

\title{
Correction: Kanduc D, et al. From Viral \\ Infections to Autistic Neurodevelopmental \\ Disorders via Cross-Reactivity. J Psychiatry Brain \\ Sci. 2018; 3(6): 14
}

The Journal of Psychiatry and Brain Science Editorial Office

There is a format error in the peptide sequences shared between viruses and human proteins in Table 4 of the published paper [1]. The corrected Table 4 can be found below. The publisher apologizes for this error.

\section{G Open Access}

Received: 29 December 2018

Accepted: 04 January 2019

Published: 09 January 2019

Copyright (C) 2019 by the author(s). Licensee Hapres, London, United Kingdom. This is an open access article distributed under the terms and conditions of $\underline{\text { Creative Commons Attribution }}$ 4.0 International License. 
Table 4. Immunopositive Epitopes Containing Sequences Shared between B19, BDV, RUBV, MeV, Influenza A Virus, and MuV Proteomes and ASD-Related or Y-Linked Proteins.

\begin{tabular}{|c|c|c|c|c|c|}
\hline IEDB ID ${ }^{1}$ & EPITOPES $^{2}$ & IEDB I $^{1}$ & EPITOPES $^{2}$ & IEDB ID $^{1}$ & EPITOPES $^{2}$ \\
\hline 1848 & aiakledakELLESS & 87732 & QTPAPKpsrappQQPQPPrmqtgr & 437698 & gpgripPРPРAPy \\
\hline 4375 & asdvetaeggeihELLRLQ & 87776 & ragltagasqsrRPRPPR & 438262 & iplPPPPAPety \\
\hline 4376 & asdvetaeggeihELLRLQsr & 97279 & eqmAGSSEQaaeameia & 442782 & arGAALALllfg \\
\hline 11286 & edakELLESSdqilr & 97289 & etyvlsiipsgpLKAEIAqkledvfagkn & 451565 & AAAPAPapa \\
\hline 12901 & ekvtgtdlelIQILKDhyni & 97293 & evlTGNLQTlkirvhegyeeftmvgrratailr & 451566 & AAAPAPpaa \\
\hline 13126 & elKLAALChgedsit & 97452 & lKNDLLEnlq & 452570 & apAAAPAPa \\
\hline 14641 & EVDGDVklssnlvil & 97554 & pLKAEIAqrledvfagk & 461930 & aEVDGDVkl \\
\hline 19737 & gggagaggaGAGGGGr & 97694 & tlelrsgywairTRSGGN & 462034 & grLVPQVRvid \\
\hline 20971 & gllaccakclyyLRGAIApr & 97740 & vlasttakameqmAGSSEQa & 470109 & RPRPPRpepppglm \\
\hline 20972 & gllacsakclyyLRGAIApr & 97766 & wairTRSGGNtnqqrasa & 470110 & RPRPPRpepppglma \\
\hline 21686 & gpLKAEIAqrle & 97779 & ymlERELVRktrflpva & 476328 & atlpsSPGLLR \\
\hline 21912 & gqlsdhphALSSSSShaepr & 106084 & rprspsSQSSSSgspprrp & 489419 & yLLKKLLql \\
\hline 22542 & gstkscarTLVSGSf & 119022 & sSQSSSSgspprrpppgrrpffhpvgeadyfeyhqe & 504031 & prspsSQSSSSgspprrppp \\
\hline 35591 & lelrsrywairTRSGGNtnqqras & 119788 & cPLSLAAqld & 507907 & RPRPPRpldshl \\
\hline 36538 & ligllaiagiRLHRAAIytaeihk & 120221 & ssssagggggGAGGGGggggsgg & 508324 & spKGASVSi \\
\hline 37232 & LLEVVQsdqsqsgltfasr & 127270 & tyvlsivpsgpLKAEIAqrl & 509573 & eAAAPAPtv \\
\hline 37300 & llfsllgLSSSSSis & 127692 & ifkiekGKVTKSielna & 509875 & lpLSSSSSv \\
\hline 39061 & IrdpisaeISIQALS & 128243 & AGSSEQaaeamevasqa & 517071 & gPGAPAGaqpaqpp \\
\hline 40581 & lvsgsfgnrfILSQGNli & 128743 & glKNDLLEnlqayqkrm & 517072 & gPGAPAGaqpaqpps \\
\hline 40826 & lyksnhnnVYWLTIp & 129032 & kameqmAGSSEQaaeam & 534817 & yivtdqkPLSLAA \\
\hline 43219 & nalypMSPLLQeclr & 129920 & sivpsgpLKAEIAqrle & 534818 & yivtdqkPLSLAAg \\
\hline 46150 & ntlelrsrywairTRSGGNt & 130187 & vaymlERELVRktrflp & 540558 & ptILSQGNrfcapder \\
\hline 48376 & pLKAEIAqrledv & 131276 & wheaqpSPGLLR & 540650 & yaiggsasptILSQGN \\
\hline 48856 & ppppeerqetrsQTPAPKps & 143551 & stlelrsrywairTRSGGNt & 540949 & agglggGAGGGGdhad \\
\hline 52333 & qshgqlsdhphALSSSSSha & 145868 & lelrsrywairTRSGGNt & 544452 & ppgapsapAAAPAPaa \\
\hline 52523 & qtgRGGSAPrpelgpptn & 150995 & hLGKCNIagwilgnp & 549187 & llqEEEEEL \\
\hline 52588 & QTPAPKpsrappQQPQPPrmqtgrg & 164390 & sstglKNDLLEnlqayqk & 552594 & htkLSSSSSitltlp \\
\hline 53963 & rgrgrgekrprspsSQSSSS & 170345 & anptILSQGNrf & 562078 & aLLEVVQsggkniel \\
\hline 54638 & RLLDRLVRl & 173446 & tILSQGNrfhap & 569127 & grLKAEIAr \\
\hline 54946 & rmqtgRGGSAPrpelgpptnpfqaava & 179908 & smDAAVAAl & 571902 & eELLRLQql \\
\hline 55937 & rsQTPAPKpsrappQQPQPPrmqt & 181520 & ynpytrsIQILKD & 574167 & kLLEVVQpcl \\
\hline 56752 & saeISIQALSyalgg & 182182 & irlradTLMGAElaarpeyr & 592219 & gdpeeeeEEEEELvd \\
\hline 58175 & sgpLKAEIAqkledvfagkn & 188697 & klcklrgvaplhLGKCNIag & 593877 & aaGAALALalw \\
\hline 58176 & sgpLKAEIAqrle & 202816 & ataAVTAAVk & 594430 & assppagpPPPPAPalvg \\
\hline 58177 & sgpLKAEIAqrledv & 227311 & krprspsSQSSSSgs & 599581 & Ltvlvprvw \\
\hline 59546 & slvgidpfkLLQNSQvyslirp & 227587 & sSQSSSSgspprrpp & 601005 & rLRELGSlvw \\
\hline 59548 & slvgidpfrLLQNSQvfsli & 239959 & laGAGGGGaavtv & 601256 & rsvsSQSSSSvs \\
\hline 60889 & ssaglKNDLLEnlqayqkrm & 245808 & aakapapkAAAPAPk & 614836 & dsSSVASKv \\
\hline
\end{tabular}


Table 4. Cont.

\begin{tabular}{cccccc}
\hline IEDB ID $^{\mathbf{1}}$ & EPITOPES $^{2}$ & IEDB ID $^{\mathbf{1}}$ & EPITOPES $^{2}$ & IEDB ID $^{\mathbf{1}}$ & EPITOPES $^{\mathbf{2}}$ \\
\hline 63973 & tgtdleIIQILKDhynisld & 252233 & akapapkAAAPAPka & 620205 & kSLLIGVfk \\
67496 & tyvlsiipsgpLKAEIAqrl & 255133 & apapkAAAPAPkaaa & 621015 & LELSEAvlptmta \\
72315 & wdleatgACICEIpt & 255442 & apkAAAPAPkaaaaa & 629659 & avPPPPAPl \\
79398 & srappppeerqesrsQTPAPKpsrapp & 316384 & kapapkAAAPAPkaa & 632869 & iSLIPPEerw \\
79399 & srappQQPQPPrmqtgRGGSAPrpelg & 348636 & papkAAAPAPkaaaa & 638702 & vpvAVTAAV \\
79544 & fapwdleatgACICEIptdv & 419951 & marGAALAL & 641923 & dyfkdlcGPDAGPig \\
79784 & dpfrLLQNSQvys & 434859 & arGAALALllf & 645191 & iqriplPPPPAPety \\
79844 & gpLKAEIAqrled & 434943 & aRTLLAKnl & 650955 & tpkdqfiiayggLRGAIA \\
\hline
\end{tabular}

${ }^{1}$ Epitopes listed according to the IEDB ID number. ${ }^{2}$ Peptide sequences shared between viruses and human proteins in capital letters.

\section{REFERENCES}

1. Kanduc D, Polito A. From Viral Infections to Autistic Neurodevelopmental Disorders via Cross-Reactivity. J Psychiatry Brain Sci. 2018;3(6):14.

How to cite this article:

The Journal of Psychiatry and Brain Science Editorial Office. Correction: Kanduc D, et al. From Viral Infections to Autistic Neurodevelopmental Disorders via Cross-Reactivity. J Psychiatry Brain Sci. 2018; 3(6): 14. J Psychiatry Brain Sci. 2019;4:e190002. https://doi.org/10.20900/jpbs.20190002 